\title{
MedienPädagogik
}

Zeitschrift für Theorie und Praxis der Medienbildung

Themenheft Nr. 36: Teilhabe in einer durch digitale Medien geprägten Welt Perspektiven des wissenschaftlichen Nachwuchses

Herausgegeben von Tim Riplinger, Jan Hellriegel und Ricarda Bolten

\section{Digitale Medien im Studium: Medienkompetent Präsentieren}

\author{
Ein Online-Kurs zum Mit- und Nachmachen \\ David Lohner, Thomas May und Adnan Seithe
}

\section{Zusammenfassung}

Am Zentrum für Mediales Lernen des Karlsruher Instituts für Technologie wird eine «akademische digitale Medienkompetenz» bei Studierenden gefördert. Verschiedene Kompetenzdimensionen werden den Lernzielen einzelner Lehrveranstaltungen zugeordnet, um eine möglichst umfassende Kompetenz zu erreichen, die Absolventen dazu befähigt, die digitale Transformation aktiv mitzugestalten. Ein erster Kurs soll als Open Educational Ressource zur Verfügung gestellt werden. So können die Inhalte künftig integrativ in alle Studiengangscurricula Einzug halten.

Digital Media in Higher Education: Presenting with Media Literacy. An online course allowing to participate and recreate

\begin{abstract}
The Center for Technology-Enhanced Learning at the Karlsruhe Institute of Technology promotes an "academic digital media literacy» among students. Different competence dimensions are assigned to the learning objectives of individual courses in order to achieve the most comprehensive competence possible, enabling graduates to actively participate in shaping the digital transformation. A first course is to be made available as an open educational resource. This will enable content to be integrated into all course curricula in the future.
\end{abstract}

\section{Kontext}

Die Digitalisierung von Gesellschaft und Arbeitswelt ist ein hochgradig dynamischer Prozess. Diesem Prozess unterliegt - wenn auch mit einer gewissen Trägheit - die Digitalität der Hochschule. Das MedienLabor ${ }^{1}$ am Zentrum für Mediales Lernen (ZML) des Karlsruher Instituts für Technologie (KIT) befasst sich auf der Metaebene mit dem Phänomen der Digitalisierung und rückt dabei die Studierenden des KIT

1 https://www.zml.kit.edu/medienlabor.php 
in den Fokus. Die Arbeitsgruppe des MedienLabors hat sich zum Ziel gesetzt, Studierende bei der Entwicklung einer digitalen Medienkompetenz zu unterstützen, die speziell das akademische Arbeitsfeld bedient, auf das ein Hochschulstudium hinarbeitet. Zahlreiche Aktivitäten des MedienLabors zielen daher darauf ab, die Ausbildung einer akademischen digitalen Medienkompetenz zu fördern. Diese Kompetenz ist nach dem Abschluss eines Hochschulstudiums im akademischen Arbeitsumfeld in der Wissenschaft von essenzieller Bedeutung, um die digitale Transformation (der Hochschul(lehr)e) mitzugestalten und daher für die Teilhabe am akademischen Leben unverzichtbar.

\section{Ausgangslage}

Um die Aktivitäten des MedienLabors und den Rahmen des Kurses «Medienkompetent Präsentieren» zu erläutern, ist eine Hinführung aus zwei Perspektiven von Nöten. Zum einen muss geklärt werden, mit welchem Verständnis von «Medienkompetenz» gearbeitet wird und zum anderen müssen die am KIT herrschenden Vorbedingungen für die Gestaltung von Lehre im besonderen Hinblick auf die interfakultative Natur des ZML dargelegt werden.

\section{Zum Kompetenzbegriff}

Wird an der Hochschule von Medienkompetenz oder «Digitaler Kompetenz» gesprochen, geschieht das häufig im Zusammenhang mit E-Learning, also aus Sicht der Lehre und der Dozierenden. In der Vergangenheit wurde hierfür der Begriff der E-Kompetenz, im Sinne einer Ein- und Durchführung von E-Learning bzw. mediengestützter Lehre geprägt (Schulmeister 2005). Der Diskurs wird mehr über Einzelanwendungen, Dienste oder bestimmte Formate geführt, denn über ein umfassendes und an der akademischen Realität orientiertes Prinzip. Einen ersten Ansatz, was Medienkompetenz bei Hochschullehrenden im Rahmen des akademischen Umfeldes ausmacht, findet sich in der folgenden Aussage bei Wedekind:

«Im akademischen Umfeld beinhaltet Medienkompetenz die Fähigkeit von Hochschullehrenden und Lernenden zum kompetenten, verantwortungsvollen und reflektierten Umgang mit digitalen Medien in den unterschiedlichen akademischen Tätigkeitsfeldern von Forschung und Anwendung, Lehre und Entwicklung. Sie umfasst darüber hinaus auch die Urteilungsfähigkeit über die (Aus-) Wirkungen des Einsatzes moderner luK-Technologien. Hochschullehrende benötigen dazu Kenntnisse, Fähigkeiten und Fertigkeiten des computergestützten und netzbasierten Präsentierens sowie multimedial und telemedial gestützten Lehrens und Lernens.» (Wedekind 2008, 5) 
Mit dieser Aussage wird die Medienkompetenz Dozierender innerhalb der alltäglichen Tätigkeitsfelder des akademischen Betriebs betrachtet und erhält so den Stellenwert einer Handlungskompetenz. Auch Reinmann et al. (2014) schlagen vor, die Bereiche des Lernens (Lehre), des wissenschaftlichen Arbeitens (Forschung) und der Organisation rund um die Hochschultätigkeit (akademische Selbstverwaltung) gleichermassen in der Formulierung einer akademischen Medienkompetenz zu berücksichtigen. Im Gegensatz zu Wedekind wird hier ergänzt, dass auch der Fokus auf die Studierenden gelegt werden muss, die selbständig lernen, forschen und ihr Studium verwalten müssen.

Wie für Dozierende muss die akademische Medienkompetenz auch für Studierende erreichbar sein, denn auch sie bewegen sich während des Studiums, aber auch - und insbesondere bei der Wahl einer Karriere in der Wissenschaft - nach dem Studium weiter im universitären Umfeld.

Bezogen auf die «digitalen» Kompetenzen Studierender kann ein weiteres Modell herangezogen werden: In ihrem Jahresbericht weisen der Stifterverband der deutschen Wissenschaft und McKinsey \& Company, Inc. (2016) darauf hin, dass aus Sicht der deutschen Arbeitgeber Hochschulabsolventen zukünftig ein digitales Kompetenzprofil aufweisen müssen, das sie für eine «Arbeitswelt 4.0» qualifiziert. Das Modell definiert hierzu drei Kompetenzdimensionen, die im Studium erworben werden: Fachkompetenzen, Berufsorientierte Kompetenzen und Persönlichkeitsbildende Kompetenzen.

An die Hochschulen erfolgt die Aufforderung, die Vermittlung zumindest digitaler Fachkompetenzen und berufsorientierter Digitalkompetenzen in den Curricula zu verankern. Persönliche Digitalkompetenzen wie z.B. kollaboratives Lernen seien zu fördern (Stifterverband für die Deutsche Wissenschaft e.V. und McKinsey \& Company, Inc. 2016, 26). Mit Blick auf die Studierenden wird der Begriff der akademischen Medienkompetenz bislang wenig betrachtet. Die mehr oder weniger intensive Nutzung digitaler Medien wird oft bereits als Indiz für einem kompetenten Umgang gesehen und damit gleichgesetzt. Dass der «Mythos der Digital Natives» nicht haltbar ist, haben Schulmeister und Loviscach (2017) ausgeführt. Die Betrachtung digitaler Kompetenzen zukünftiger Absolventen muss vor dem Hintergrund des klar umrissenen akademischen Arbeitsfelds aus Lehre/Lernen, Forschung und akademischer Selbstverwaltung in wissenschaftlichen Einrichtungen wie Hochschule und Universität stattfinden. Es gilt, die im privaten Bereich ausgebildeten Kompetenzen im Umgang mit digitalen Medien, Diensten und Tools (vgl. hierzu Schulmeister 2008; Calmbach u.a. 2016) zu einer akademischen Medienkompetenz weiter zu entwickeln.

Ein auf weiteren Kompetenzmodellen basierendes Kompetenzraster zur Messung von digitaler Kompetenz von Hochschullehrenden haben Eichhorn et al. (2017a) entworfen und validiert (Eichhorn und Tillmann 2018). Die Autoren verwenden acht Kompetenzdimensionen auf drei Stufen. Die Dimensionen orientieren sich dabei am 
Digital-Literacy-Framework (JISC 2014), die Stufen an Blooms Lernzieltaxonomie (Bloom, Engelhart, und Füner 1973). Für die Arbeit am MedienLabor mit Fokus auf den Studierenden sollte das Modell mit geringer Veränderung (vgl. Abb. 1) anwendbar sein. Hierzu wird die Dimension «Digitale Lehre» umbenannt in «Digitales Lernen» um sie in die studentische Perspektive zu bringen. Auf diese Weise rückt die Bedeutung des Digitalen für den eigenen Lernprozess in den Mittelpunkt. Die einzelnen «Kann-Beschreibungen» aller Dimensionen (Eichhorn, Müller, und Tillmann 2017b), die die Kompetenzen näher beschreiben, werden entsprechend angepasst.

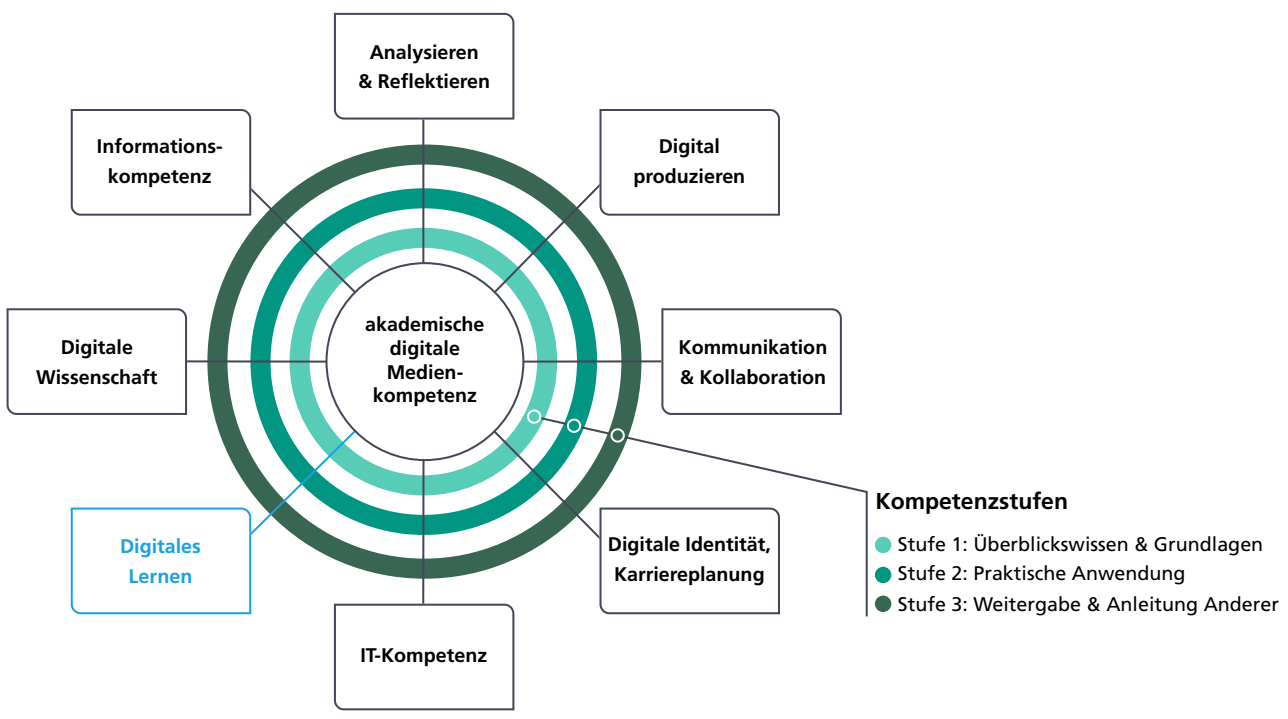

Abb. 1.: Acht Kompetenzdimensionen einer akademischen digitalen Medienkompetenz nach Eichhorn et al. (2017). Verändert durch das ZML MedienLabor (umbenannte Dimension «Digitales Lernen»).

Auf diese Weise ergibt sich ein umfassendes Kompetenzraster, an denen sich Lehrveranstaltungen, die explizit die akademische Medienkompetenz fördern sollen orientieren können. Ziel des MedienLabors ist es, Lehrveranstaltungen so auszurichten, dass alle Kompetenzdimensionen in allen Stufen vermittelt werden. Es liegt auf der Hand, dass eine umfassende akademische Medienkompetenz unmöglich in nur einer solchen Lehrveranstaltung entwickelt werden kann. Gleichzeitig ist es notwendig, diese Kompetenz zu entwickeln, um als aktives Mitglied der Gesellschaft an Prozessen partizipieren zu können und die zukünftige (akademische Arbeits-) Welt zu mitzugestalten. Diese umfassende Betrachtung digitaler Phänomene in mehreren Kompetenzdimensionen soll Studierende dazu befähigen, das akademische Feld multiperspektivisch zu betrachten. Mit dem so gewonnenen erweiterten Reflexionsvermögen können sie ihr eigenes Handeln und dessen Auswirkungen im Kontext des Wissenschafts- und Lehrbetriebs evaluieren und eine durch Medien geprägte Welt konstruktiv mitgestalten. 


\section{Zum Lehrkonzept des MedienLabors}

Das MedienLabor des KIT ist am Zentrum für Mediales Lernen (ZML) verortet und damit Teil einer interfakultativen Einrichtung, die keinen eigenen Studiengang anbietet. Daher finden alle Lehrveranstaltungen des MedienLabors innerhalb des sogenannten Schlüsselqualifikationsangebots (SQ-Angebot) oder an kooperierenden Instituten des KIT statt. Ziel der Lehre des MedienLabors ist es, Studierende bei der Entwicklung einer akademischen digitalen Medienkompetenz zu unterstützen und sie damit auf eine zunehmend digitalisierte akademische Arbeitswelt vorzubereiten. Auf Basis etablierter Modelle zur Beschreibung einer digitalen Kompetenz (s.o.) werden in explorativen Settings Studierende zum gezielten Transfer ihres individuellen, hauptsächlich privat geprägten Medienverhaltens (siehe hierzu White und Le Cornu 2011) in Ihre Lebenswelt des Studiums angehalten. Durch dieses Bewusstmachen wird die reale Nutzung bestehender persönlicher Kompetenzen in einen wissenschaftlichen Kontext übertragen. Dabei wird stets auf eine sinnvolle Kombination von theoretischer Fundierung und praktischem Exkurs gesetzt. In Individual- oder Team-Challenges setzen sich Studierende gezielt problemorientiert mit Themenfeldern (Komponenten) einzelner Dimensionen praxisnah auseinander und reflektieren diese vor ihrem eigenen Fachstudium. Im Sinne des Forschenden Lernens entwickeln die Studierenden ihre Umsetzung selbst und wählen selbst geeignete Methoden und Werkzeuge für die gegebenen Anforderungen, die Bewertung und Darstellung der Ergebnisse aus. Dabei ist es wichtig, die Alltäglichkeit, aber auch Besonderheit des Medienhandelns vor allem der Forschung aufzuzeigen (Reinmann 2015). Mit dieser Methode haben die Studierenden die Chance, Teile der Lehrveranstaltung bzw. ihren Leistungsnachweis massgeblich selbst mitzugestalten. Dadurch ermöglicht das MedienLabor eine hohe Selbstverpflichtung, die eine intrinsische Motivation, sich weiter mit den Themen zu befassen, fördern kann.

\section{Lehre am MedienLabor}

Im Sommersemester 2019 bietet das MedienLabor vier Lehrveranstaltungen an, von denen drei über das SQ-Angebot creditfähig sind. Alle Kurse haben online-Anteile, um bereits im Kursformat selbst digitale Kompetenzen zu fördern:

1. Einführung in LaTeX: Dieser nicht-creditfähige Kurs ist ein Onlineangebot über die Textsatzumgebung LaTeX mit besonderem Fokus auf dem Einsatz von mathematischen Formeln. Der Kurs ist nicht betreut und findet ausschliesslich online im Selbststudium statt.

2. Digitale akademische Medienkompetenz: Dieser Kurs befasst sich mit den Kompetenzmodellen und ihrer Relevanz für die Studierenden jeweils im Kontext ihres eigenen Studiums. Der Kurs findet im Blended-Learning Format statt. 
3. Schnelle \#wisskomm mit Videos: Dieser Kurs ist eine Übung im Studiengang Wissenschaftskommunikation eines kooperierenden Instituts und behandelt die Videoproduktion mit mobilen Endgeräten zur Wissenschaftskommunikation und findet als Flipped Classroom statt.

4. Medienkompetent Präsentieren: Dieser Kurs befasst sich mit der digital-medialen (und technischen) Gestaltung von Präsentationen. Dabei liegt der Fokus auf der kritischen Bewertung konventioneller Formate und dem explorativen Erproben neuer Möglichkeiten. Im Flipped Classroom Format gibt es ausreichend Raum für einen experimentellen Zugang zum Thema.

Legt man die acht Kompetenzdimensionen von Eichhorn et al. an die Kurse an, so sind die Dimensionen «Bedienen und Anwenden», «Produzieren/Präsentieren» und «Analysieren/Reflektieren» bereits gut (bis auf Stufe zwei) vertreten. Die Dimensionen «Digital informieren/recherchieren» und «digital kommunizieren/kooperieren» sind bislang auf Stufe eins einzuordnen. "Digitale Wissenschaft» und die vom MedienLabor umbenannte Dimension «Digitales Lernen» werden über die Kursformate selbst und die Erbringung des Leistungsnachweises gefördert. Damit deckt das MedienLabor bisher sieben der acht Dimensionen ab, wenn auch noch ausbaufähig. Die Dimension «digitale Identität und Karriereplanung» findet bislang keine Berücksichtigung im Kursangebot.

\section{«Medienkompetent Präsentieren» im Detail}

Am KIT gibt es im entsprechenden SQ-Bereich zahlreiche Kurse, die die Präsentations- und Kommunikationsfähigkeiten der Studierenden thematisieren ${ }^{2}$. Bis zum Wintersemester 2017/18 hat sich keiner der Kurse explizit mit der Handhabung, Eignung und Bewertung der digital-medialen Werkzeuge, die für eine Präsentation nötig sind (sowohl Hard- als auch Softwaretools) befasst. Der neu konzipierte SQ-Kurs «Digitale Medien im Studium: Medienkompetent präsentieren» schliesst diese Lücke zwischen theoretischem Wissen, praktischer Handlungsfähigkeit und kritischem Reflexionsvermögen in Bezug auf digitale Präsentationen. Die Schnittstelle zwischen visueller Kommunikation einerseits und «digitalem Handwerkszeug» andererseits bietet vielfältige Ansatzpunkte für den Erwerb von Medienkompetenz. Der Kurs knüpft an der Lebenswelt von Studierenden an, die jedes Semester mehrere Referate, bzw. Vorträge halten müssen und die in ihrer beruflichen Zukunft Ideen vor Teammitgliedern präsentieren und verteidigen müssen. Nachdem der Kurs zunächst als «Proof of Concept» im Wintersemester 2017/18 als klassische Präsenzveranstaltung gehalten wurde, wird er im Sommersemester 2018 als Flipped Classroom mit hohem OnlineAnteil umgesetzt, die meist diesem Schema folgen:

2 https://studium.hoc.kit.edu/index.php/2018/07/03/praesentieren-kommunizieren 
- Unter den Onlinematerialien sind kurze Lehr- und Impulsvideos zu den Themen, die im Kurs aufgegriffen werden; Links zu weiteren Videos und Webseiten, die unterschiedliche Präsentationstools vorstellen und «Steckbriefe» über die jeweils vorgestellten Tools mit relevanten Informationen für Studierende (z.B. Bezugsquellen, Preise, Eignung für (fach-) spezifische Anforderungen).

- Die oben genannte Lücke zwischen reflektiertem Wissen und praktischem Handeln schliesst der Kurs in Bezug auf Präsentationen, indem digital-mediale Werkzeuge explizit thematisiert werden: Zunächst werden Mainstream- und NischenProdukte (Hard- und Software sowie cloudbasierte Dienste) in kurzen Referaten in den Präsenzveranstaltungen vorgestellt und Erfahrungen unter den Studierenden ausgetauscht und kritisch beleuchtet.

- Anschliessend werden die Tools explorativ erprobt und bewertet. Dabei spielt der individuelle Kontext der Studierenden eine grosse Rolle, die während des Semesters ihr neu erworbenes Wissen durch Beobachtungen aus ihrem eigenen Studienalltag erweitern. Dabei muss stets zwischen Aufwand-Nutzen und Vorgaben der Hochschule abgewogen werden.

Mit diesem Kurskonzept findet eine kontinuierliche Auseinandersetzung mit der wechselseitigen Beziehung von Hard- und Softwaretools mit den jeweiligen Spezifika der einzelnen Studienfächer statt. Es werden aktuelle Meinungen und Vorlieben in Bezug auf die technische und ästhetische Gestaltung von Präsentationen diskutiert. Im Studierendenalltag kommt dieser Diskurs viel zu kurz, sofern er überhaupt stattfindet. In Zusammenarbeit mit der Nachwuchsforschungsgruppe «Science in Presentations» des $\mathrm{KIT}^{3}$ beleuchten weitere Beiträge das Themenfeld aus der wissenschaftlichen Perspektive der Kommunikationsforschung.

\section{Beispiel einer Lerneinheit}

Im konkreten Fall des Kurses «Medienkompetent Präsentieren» soll der typische Ablauf der Lerneinheiten hier kurz dargestellt werden. Dabei wird die Lehrmethode des expositorischen Lernens verwendet (Ausubel 1963; zitiert nach Klauer und Leutner 2012, 153), mit der Lernende neues Wissen in bereits bestehende Wissensstrukturen einordnen können.

In den Einheiten geht es darum, den Studierenden Hard- und Software nahezubringen, die ihnen helfen, eine Bildschirmpräsentation mit einschlägigen Programmen souverän zu steuern.

- Onlinephase (Input): Ein kurzes Video zeigt verschiedene Hardwareprodukte unterschiedlicher Art gezeigt und deren Eigenschaften und Einsatzmöglichkeiten für eine Präsentation wird kurz erläutert: Presenter, Remote-Apps

3 http://www.geistsoz.kit.edu/germanistik/2493.php 
für Smartphones, Funkmaus, Funktastaturen mit eingebauten Trackpads. Ein weiteres Video demonstriert die Referentenansicht (Microsoft PowerPoint) bzw. die Moderationsansicht (Apple Keynote) bzw. das Äquivalent von LibreOffice und deckt Unterschiede in der Funktionsweise auf.

- Onlinephase (Aktivität): Die Lerneinheiten werden von Aufgaben begleitet, in denen die Studierenden aufgefordert sind, ihr eigenes Präsentationsverhalten auf diese Steuerungsmöglichkeiten zu reflektieren und sowohl diese Selbstreflexion als auch Beobachtungen in ihrem studentischen Umfeld zu bewerten.

- Präsenzphase: Gemeinsam mit allen Kursteilnehmern und dem Dozierenden werden alle Optionen explorativ getestet, evaluiert und besprochen werden.

\section{Pilot für einen offenen Kurs}

Der Kurs «Medienkompetent Präsentieren» stellt einen Piloten dar: Der Kurs soll als Open Educational Ressource (OER) freigegeben werden, um das Konzept leicht an jeden (Hochschul-)Standort zu transferieren. Ausserdem kann der Kurs von mehreren Parteien weiterbearbeitet und ergänzt werden. Auf diese Weise können fachspezifische Eigenheiten und eine viel grössere Anzahl an Tools und Themen abgedeckt werden. Perspektivisch entsteht möglicherweise ein Pool an Lerneinheiten zu bestimmten Themen, aus denen sich dann wiederum neue Kurse generieren lassen, die jeweils leicht andere Schwerpunkte setzen.

Dafür hat das MedienLabor darauf geachtet, dass die erstellten Materialien (Videos und Präsentationen) unter offenen Lizenzen (Creative Commons Namensnennung; (C-BY) stehen und eine möglichst gut und unkompliziert weiterverwendbar sind. Darüber hinaus bedient sich der Kurs zahlreichen Elementen, die öffentlich im Internet-vornehmlich auf YouTube-zur Verfügung stehen. Die Kursinhalte sind in H5P-Bausteinen organisiert, sodass sie sich in gängige Learning Management Systeme einbinden lassen und bei Bedarf editierbar sind. Auf diese Weise ermöglicht der Kurs Interessierten einerseits am Kurs teilzunehmen, andererseits auch, ihn an die eigenen Bedürfnisse anzupassen, zu verändern oder weiterzuentwickeln. Es stehen noch rechtliche Fragen aus, bis der Kurs oder die einzelnen Elemente veröffentlicht werden können. Es ist geplant, die Bausteine des Kurses einzeln und als Paket zur Verfügung zu stellen. So kann der Kurs als Ganzes verwendet werden oder nur die Teile, die für Interessierte besonders relevant sind.

\section{Nächste Schritte und offene Fragen}

Bis die rechtlichen Rahmenbedingungen geklärt sind, erstellt das MedienLabor Begleitmaterial zum Kurs «Medienkompetent Präsentieren», mit dessen Hilfe eine Durchführung ausserhalb des KIT geplant werden können. Darin werden die einzelnen 
Kursbausteine erläutert, Lernziele aufgelistet, Methoden zu deren Erreichung dargestellt und einige inhaltliche Aspekte aufgegriffen, da davon auszugehen ist, dass die Kursinhalte auf das individuelle Vorwissen derer angepasst werden muss, die den Kurs durchführen wollen. Ausserdem müssen für eine modulare Bereitstellung auf dieser Ebene die zu erreichenden Kompetenzdimensionen den einzelnen Aufgaben zugeordnet werden.

Da es sich beim Kurs «Digitale Medien im Studium: Medienkompetent Präsentieren» wie beiden anderen Kursen des MedienLabors derzeit um ein freiwilliges SQ-Angebot handelt, steht die Frage im Raum, wie künftig sichergestellt werden kann, dass eine akademische digitale Medienkompetenz in jedem Studiengangscurriculum des KIT integriert wird. Das MedienLabor am ZML hat sich langfristig zum Ziel gesetzt, seine Angebote ganz oder teilweise in den Modulhandbücher einzelner Studiengänge zu platzieren. Der eingeschlagene Weg, Inhalte als OER bereitzustellen kann dabei helfen, die Integration leichter zu gestalten, ist gleichzeitig aber nur eine von vielen Möglichkeiten, Studierende auf eine von digitalen Medien geprägte Welt vorzubereiten.

\section{Literatur}

Ausubel, David G. 1963. «Cognitive Structure and the Facilitation of Meaningful Verbal Learning1». Journal of Teacher Education 14 (2): 217-22. https://doi.org/10.1177/002248716301400220.

Bloom, Benjamin Samuel, Max D Engelhart, und Eugen Füner. 1973. Taxonomie von Lernzielen im kognitiven Bereich. Beltz Weinheim, Germany.

Calmbach, Marc, Silke Borgstedt, Inga Borchard, Peter Martin Thomas, und Berthold Bodo Flaig. 2016. Wie ticken Jugendliche 2016? Lebenswelten von Jugendlichen im Alter von 14 bis 17 Jahren in Deutschland. Wiesbaden: Springer.

Eichhorn, Michael, Ralph Müller, und Alexander Tillmann. 2017a. «Entwicklung eines Kompetenzrasters zur Erfassung der „Digitalen Kompetenz“ von Hochschullehrenden». In Bildungsräume. Proceedings der 25. Jahrestagung der Gesellschaft für Medien in der Wissenschaft, 72:209-19. Medien in der Wissenschaft. Chemnitz: Waxmann.

Eichhorn, Michael, Ralph Müller, und Alexander Tillmann. 2017b. «Kompetenzraster_DigitaleKompetenz-Hochschullehrende.pdf». Digitale Kompetenz bei Hochschullehrenden - Kompetenzbeschreibungen auf acht Dimensionen und drei Stufen. 2017. http://www.studiumdigitale.uni-frankfurt.de/65903024/Kompetenzraster_Digitale-Kompetenz-Hochschullehrende.pdf.

Eichhorn, Michael, und Alexander Tillmann. 2018. «Digitale Kompetenzen von Hochschullehrenden messen». In Die 16. E-Learning Fachtagung Informatik, herausgegeben von Detlef Krömker und Ulrik Schroeder, 69-80. Lecture Notes in Informatics (LNI). Bonn.

JISC, Joint Information Systems Commitee. 2014. «Developing Digital Literacies». Jisc. 2014. https://www.jisc.ac.uk/full-guide/developing-digital-literacies. 
Klauer, Karl Josef, und Detlev Leutner. 2012. Lehren und Lernen: Einführung in die Instruktionspsychologie. 2., überarb. Aufl. Weinheim: Beltz.

Reinmann, Gabi. 2015. «Lehren und Lernen mit Digital Natives im Kontext forschungsgeleiteter Lehre». Universität Wien.

Reinmann, Gabi, Silvia Hartung, und Alexander Florian. 2014. «Akademische Medienkompetenz im Schnittfeld von Lehren, Lernen, Forschen und Verwalten». In Grundbildung Medien in pädagogischen Studiengängen, herausgegeben von Peter Imort und Horst Niesyto, 319-32. Schriftenreihe Medienpädagogik interdisziplinär. München: kopaed.

Schulmeister, Rolf. 2005. «Welche Qualifikationen brauchen Lehrende für die „Neue Lehre“? Versuch einer Eingrenzung von eCompetence und Lehrqualifikation». In Hochschulen im digitalen Zeitalter. Innovationspotenziale und Strukturwandel. (= education quality forum 2004), herausgegeben von R. Keil-Slawik und M. Kerres, 215-34. Münster: Waxmann.

Schulmeister, Rolf. 2008. Gibt es eine Net Generation? Widerlegung einer Mystifizierung. Gesellschaft für Informatik e.V. http://dl.gi.de/handle/20.500.12116/14998.

Schulmeister, Rolf, und Jörn Loviscach. 2017. «Mythen der Digitalisierung mit Blick auf Studium und Lehre». In Digitale Transformation im Diskurs. Kritische Perspektiven auf Entwicklungen und Tendenzen im Zeitalter des Digitalen, herausgegeben von Claudia De Witt und Christian Leineweber. http://www .medien-im-diskurs.de.

Stifterverband für die Deutsche Wissenschaft e.V., und McKinsey \& Company, Inc., Hrsg. 2016. Hochschulbildung für die Arbeitswelt 4.0. Hochschul-Bildungs-Report 2020: Jahresbericht 2016. Essen.

Wedekind, Joachim. 2008. «Medienkompetenz für (Hochschul-) Lehrende». Herausgegeben von S. Seufert. Zeitschrift für E-Learning, Nr. 2: 14.

White, David S., und Alison Le Cornu. 2011. «Visitors and Residents: A new typology for online engagement». First Monday 16 (9). https://doi.org/10.5210/fm.v16i9.3171. 